\title{
Microneurography of Peripheral Nerves in Mice: An Old Approach Applied to the Novel Concept of Neural Modulation of Immune Organs in Cardiovascular Diseases
}

\author{
Lorenzo Carnevale*1, Marialuisa Perrotta ${ }^{2}$ and Giuseppe Lembo ${ }^{1,2}$ \\ ${ }^{1}$ Department of Angiocardioneurology and Translational Medicine, Italy \\ ${ }^{2}$ Department of Molecular Medicine, Sapienza University of Rome, Italy
}

Received: February 22, 2018; Published: February 28, 2018

*Corresponding author: Lorenzo Carnevale, Department of Angiocardioneurology and Translational Medicine, IRCCS Neuromed, Pozzilli (IS), Italy, Tel: 390865915225; Email: lorenzo.carnevale@neuromed.it

\begin{abstract}
Microneurography in small animals has proven to be a fundamental tool to investigate novel concepts of immune modulation by the autonomic nervous system. Major discoveries in the field pushed toward new investigation in the cardiovascular pathophysiology, to explore the relations between immune organs such as the spleen and hypertension. Given the variety of nerves of interest a great effort has to be put in the standardization of technical, experimental setup and analysis procedures.
\end{abstract}

Keywords: Microneurography; Splenic Nerve Recording; Neuroimmune Modulation; Sympathetic Nerve Recording

\section{Introduction}

Understanding how the central nervous system (CNS) controls the different organs is a critical task in physiology since the first pioneering studies [1]. Evidenced the technological capabilities to directly record the human peripheral nerve activity. With the evolution of the experimental research models through genetic engineering the focus of pre-clinical studies pointed toward the study of specific genetic alterations induced primarily in small animals, such as mice and rats. At the same time the innovation in technical equipment and the improved miniaturization of acquisition devices paved the way for electrophysiological recording in such animal models. Through this, the researchers gained powerful tools to explore the interactions between the CNS and other organs by studying the alterations in nerve traffic recording after different stimuli or in different genetic conditions.

The core concept of this technique is to record the electrical currents flowing through a nerve, analyzing this signal by characterizing the activations (spikes) or groups of activations [2]. The influence of the autonomic nervous system in cardiovascular pathophysiology has been extensively investigated through studies in hypertensive animals and patients, showing an elevated sympathetic nervous system outflow coupled with an enhanced vascular reactivity $[3,4]$. The microneurography played a pivotal role in this branch of research, focusing mainly on the skeletal muscle in humans and on the renal district in mice. The last decade marked further advancements with a major breakthrough in the field, with the discovery of the inflammatory reflex driven by a parasympathetic-sympathetic modulation of an immune organ as the spleen $[5,6]$. Although initially investigated in the context of immune and inflammatory reactions the inflammatory reflex became soon object of interest in different path physiological settings. More recently, the field of cardiovascular investigations started to pursue this direction, finding that the same pathway of neuroimmune modulation is relevant in pathophysiological context as hypertension where, thus far, the scenario was dominated by renal nerves [7].

\section{Technical Setup and Experimental Procedures}

The great heterogeneity existing between the various districts which can be recorded poses a great challenge in terms of procedures and equipment standardization [2]. The first discriminate is whether the recording is performed in conscious and unrestrained status [8]. Or in an anesthetized animal [7,9]. The former usually consist in a surgery procedure to access and expose the interested 
nerve district, and then the electrodes are placed in contact with the nerve and isolated from surrounding tissue, while a telemetry device is implanted to monitor blood pressure. The latter procedure is adopted when the nerve bundle to be recorded is too frail to be monitored continuously with conscious animal or when the surgical access to the nerve is too challenging (Figure 1A). In case of conscious animal, it is possible to achieve long periods of SNA recordings, with the necessary attention in controlling whether the signal quality remains preserved during the whole experiment [10]. Concerning the experimental technical setup, a general consensus is evidenced through the literature, even though no "gold standard" is established. The terminal part of the recording apparatus, the electrodes, is usually a pair of platinum-iridium [9]. Or stainlesssteel leads, with a third lead for the ground wire. The electrodes are connected to a pre-amplifier to set a high gain to the signal and then to a differential amplifier. The resulting signal is then acquired and sampled through software for digital filtering and further analyses. A core issue of this step is to ensure that the sampling frequency is sufficiently high to respect the Nyquist principle (to sample at least with double the frequency respect to the maximum frequency of the signal you want to analyze) to observe a clean signal without aliasing (Figure 1B).

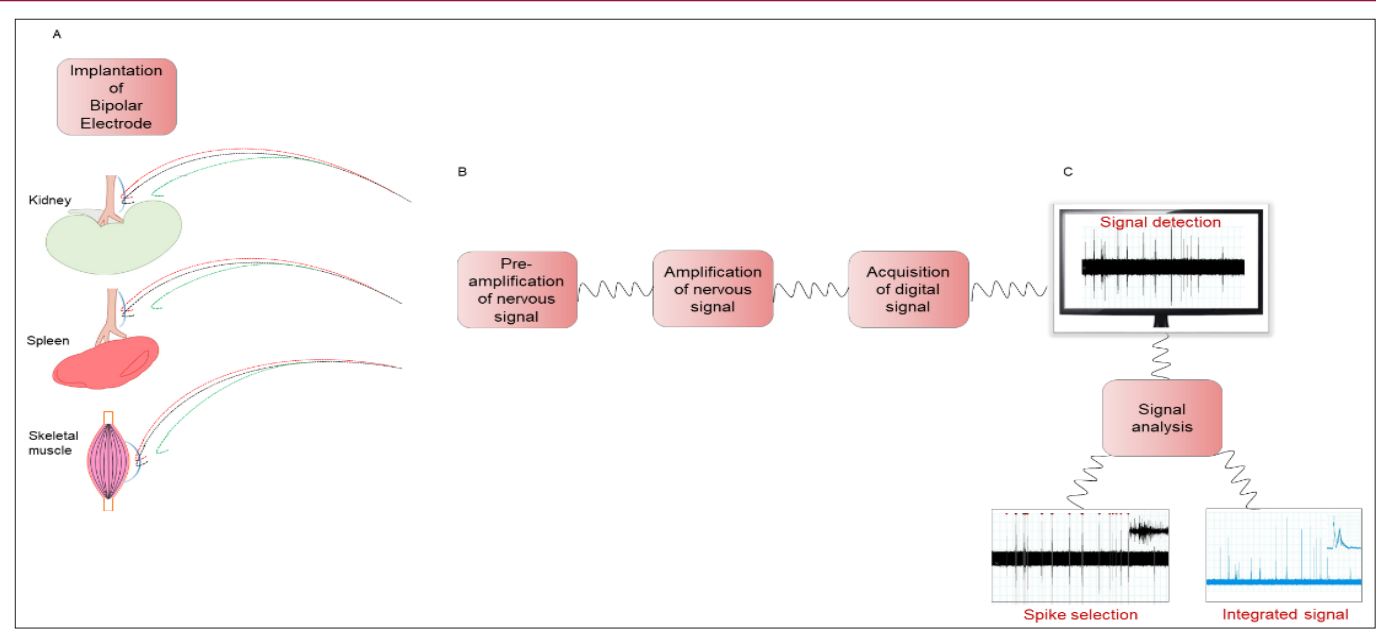

Figure 1: From target organs to the analysis of nervous signals. A) Schematic representation of the chronic electrode implantation around the peripheral nerve in target organs as kidneys, spleen and skeletal muscle. B) Box scheme of electronics technical setup. C) Detection and analysis of nervous signal by the selection of spikes and the integrated signal.

These techniques have been extensively used in experimental hypertension studies, focusing mainly on renal nerves [8]. The results showed a promising translational potential for treatment of hypertensive patients by denervation or neural modulation, even though the results obtained in clinical trials have showed no definitive result yet. To further explore the relationship between CNS and cardiovascular diseases, our group focused on a less explored district, with the aim of studying how the neural modulation of the immune system, conveyed on the spleen, is able to modulate hypertensive responses. Thus we established a procedure to access the splenic nerve in mice and record its neural activity during the exposure of the animal to hypertensive challenges, as Angiotensin II (AngII) or salt-sensitive stimuli (DOCA-salt) [7]. Once the setup procedures are completed, the recording can be carried on. To have a consistent evaluation of the nerve activity the first target is to achieve a stable baseline recording of both nerve activity and pressure parameters.

Then the system can be used to monitor acute or chronic responses. In the first case, after obtaining the baseline signal, it is possible to monitor acute responses to vasoactive stimuli can be applied, like bolus injection of sodium nitroprusside / phenylephrine to challenge the sympathetic nerve activity [8]. Motor or nasopharyngeal stimuli [11]. In the case that one is instead interested in the modulation of nerve activity upon chronic in vivo stimuli, like AngII, mice are exposed to the substance for a time window of interest and then the recording procedure is performed and nerve activity is evaluated and compared to a control group of mice, exposed to the vehicle alone. In either case, after the desired time of recording, a good practice is to obtain noise estimation by recording the nerve without activity, inducing a ganglionic blockade (i.e. hexametonium injection) or recording a post mortem track activity [7]. Completed all the recording procedures, it is possible to proceed with further analysis, to quantify the signal activity and evaluate the difference in baseline or stimuli response.

\section{Analysis}

The different characteristics of the recorded signals call for the implementation of various analysis methods and techniques. First of all, it is very common to analyze not only the raw signal, but also the integrated signal. To do this, usually a time-decay integral window is used, transforming bursts of the raw signal into single peaks on the integrated signal [12]. Once both signals are obtained, the first step is to obtain a reference noise level by quantifying the noise integral level or the raw signal noise threshold value. After this step is completed the different signals can be evaluated by alteration of the activity respect to the baseline recording, in case of stimuli response, or in absolute quantification of nerve activity in case of baseline group comparison between different chronic treatments or genetic alterations (Figure 1C). The analysis of stimuli response is usually expressed in relative 
variation respect to the baseline activity, by fixing the normal value as the mean of the integral during the baseline. Once one of the stimuli is induced in the animal, the variation of the integral value is usually expressed in percentage increase or decrease of activity. This approach is implemented to mitigate in the analysis a series of technical issues which can greatly influence the absolute values of the recordings, like the stability of nerve-electrodes connection and the amplification conditions $[9,10]$.

The analysis of chronic conditions or genetic alterations doesn't rely on the observation of a change in the signal during the recording, instead has the objective to evaluate differences in the baseline recordings by absolute quantification of nerve activity. The identification of a threshold value to distinguish between recording noise and nerve activity is a crucial task in the analysis. This value is then used as a minimum value for spike detection: the researcher can select a certain time frame in which every segment of the signal above the noise threshold is selected and counted as nerve activity. These single spikes have to be further discriminated, by excluding artefactual elements (like movement noise) by selecting spikes with certain values of duration and intensities. This procedure can give an absolute value of spikes per time frame of the baseline activity, values which can be compared between different animals and experimental groups [7]. (Figure 1C). The complexity of the signals obtained from nerve recordings calls for the implementation of novel, more sophisticated analysis methods and strategies. The analysis not only in the time domain, but also in the frequency domain can further describe the signals, adding spectral features to the characterization. Adding features to the signal description can open the analysis to the artificial intelligence field, by using automated system to classify for example artefacts from nerve activity or different kind of nerve activities.

\section{Conclusion}

Assessing peripheral nerve activity is a potent tool to study how the CNS drives the different organs functions. During the years researchers and technology elevated the technical capabilities and deepened the biological insights acquired by these studies. Even though no "gold standard" is defined in the field, the technical setup needed to perform with success this kind of analysis is consolidated through the literature. The analysis of the acquired signals is less standardized, greatly depending on which question drives the study, whether we have interest in analyzing some kind of stimuli response or baseline activity comparison. The further technological advances and computer science innovations also open up the possibility to a new evolution in the field, by the means of richer signal characterization and artificial intelligence exploitation to obtain deeper insights from data.

\section{References}

1. Hensel H, Boman KK (1960) Afferent impulses in cutaneous sensory nerves in human subjects. Journal of Neurophysiology 23(5): 564-578.

2. Stocker SD, Muntzel MS (2013) Recording sympathetic nerve activity chronically in rats: surgery techniques, assessment of nerve activity, and quantification. American Journal of Physiology-Heart and Circulatory Physiology 305(10): H1407-H1416.

3. Grassi G (2010) Sympathetic neural activity in hypertension and related diseases. American journal of hypertension 23(10): 1052-1060.

4. Malpas SC (2010) Sympathetic nervous system over activity and its role in the development of cardiovascular disease. Physiological reviews $90(2):$ 513-557.

5. Rosas-Ballina M, Ochani M, Parrish WR, Ochani K, Harris YT, et al. (2008) Splenic nerve is required for cholinergic antiinflammatory pathway control of TNF in endotoxemia. Proceedings of the National Academy of Sciences 105(31): 11008-11013.

6. Tracey KJ (2002) The inflammatory reflex. Nature 420(6917): 853-859.

7. Carnevale D, Perrotta M, Pallante F, Fardella V, Iacobucci R, et al. (2016) A cholinergic-sympathetic pathway primes immunity in hypertension and mediates brain-to-spleen communication. Nature communications 27(7): 13035.

8. Hamza SM, Hall JE (2012) Direct Recording of Renal Sympathetic Nerve Activity in Unrestrained, Conscious MiceNovelty and Significance. Hypertension 60(3): 856-864.

9. Rahmouni K, Morgan DA (2007) Hypothalamic arcuate nucleus mediates the sympathetic and arterial pressure responses to leptin. Hypertension 49(3): 647-652.

10. Guild SJ, Barrett CJ, Mc Bryde FD, Van Vliet BN, Head GA, et al. (2010) Quantifying sympathetic nerve activity: problems, pitfalls and the need for standardization. Experimental physiology 95(1): 41-50.

11. Burke SL, Head GA (2003) Method for in vivo calibration of renal sympathetic nerve activity in rabbits. Journal of neuroscience methods 127(1): 63-74.

12. Malpas SC, Ninomiya I (1992) A new approach to analysis of synchronized sympathetic nerve activity. American Journal of Physiology-Heart and Circulatory Physiology 263(4): H1311-H1317.

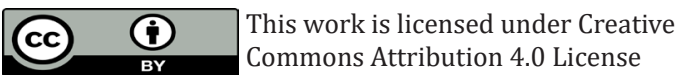

Submission Link: http://biomedres.us/submit-manuscript.php

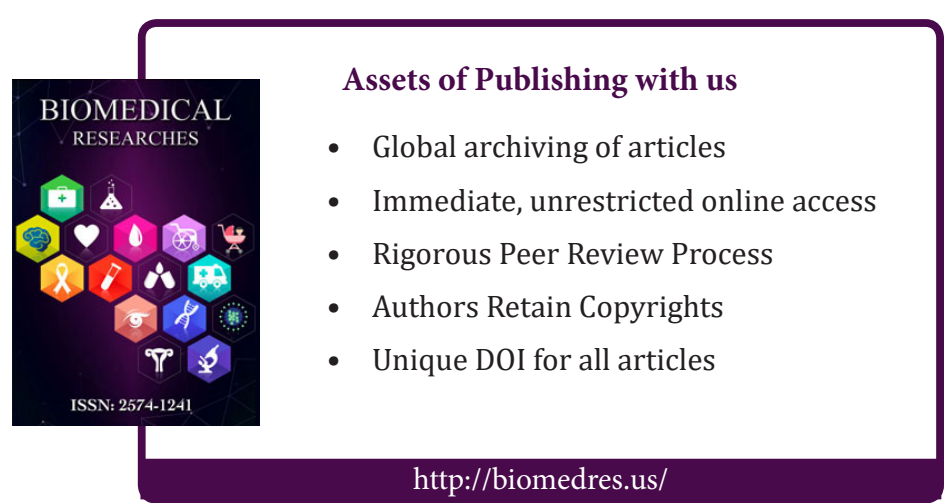

